\title{
Long-term survival after excision of a solitary esophageal cancer brain metastasis
}

Kenneth A. Kesler, MD, ${ }^{\text {a }}$ Zane T. Hammoud, MD, ${ }^{\text {a }}$ Paul R. Helft, MD, ${ }^{\text {b }}$ Karen M. Rieger, MD, ${ }^{a}$ Michael B. Pritz, MD, PhD, ${ }^{c}$ and John W. Brown, MD, ${ }^{a}$ Indianapolis, Ind

$\mathrm{L}$ ocally advanced esophageal cancer remains a disease with a low long-term cure rate, despite combined modality therapy, because of the frequent presence of occult metastatic disease. Metastatic disease to the central nervous system (CNS) is unusual, with a reported incidence of between $0.4 \%$ and $5.1 \% .{ }^{1}$ Patients with esophageal cancer who do present with CNS metastases after initial treatment represent a clinical dilemma. Although several treatment strategies using radiotherapy, surgical intervention, or both for CNS metastases exist, a poor prognosis is anticipated. We report long-term survival in a patient treated for a locally advanced adenocarcinoma of the esophagus and subsequently for a CNS metastasis.

\section{Clinical Summary}

The patient presented in June 1999 as a 53-year-old healthy white male with dysphagia and a 27-pound weight loss. Endoscopy identified a mass in the distal esophagus from 35 to $39 \mathrm{~cm}$. A biopsy was positive for poorly differentiated adenocarcinoma. No evidence of metastatic disease by means of computed tomographic (CT) scans of the chest and abdomen was found. Transesophageal ultrasonography demonstrated a transmural neoplasm with a cytologically positive celiac lymph node equating to stage IVa disease. He was treated with 4 cycles of cisplatin, 5-fluorouracil, leucovorin, and concurrent radiation (5040 cGy in 28 fractions), which he tolerated well. Repeat CT scans revealed distal esophageal thickening without interval development of metastatic disease. Six weeks after completion of chemoradiation, esophagogastrectomy with en bloc "2-field" (celiac-mediastinal) lymphadenectomy was performed through an Ivor Lewis approach. He was discharged in good condition on the 10th postoperative day. Final pathology demonstrated extensive ulceration of the distal esophagus without evidence of residual neoplasm. Of 17 lymph nodes harvested from the surgical specimen, one had pathologic evidence of necrotic (nonviable) neoplasm, whereas the remainder were found to be free of previous or active disease.

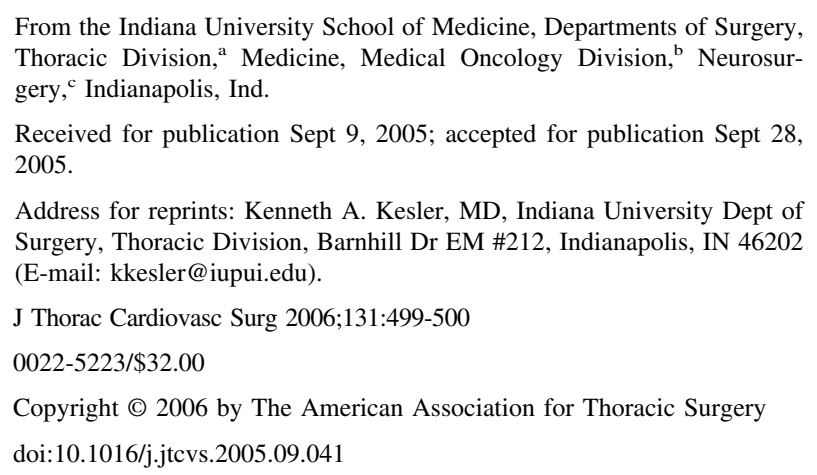

Six months after esophagogastrectomy, an acute mild right hemiparesis developed. Magnetic resonance imaging revealed a single $3-\mathrm{cm}$ enhancing mass in the left posterior frontal lobe with surrounding edema (Figure $1, A$ and $B$ ). No other evidence of recurrent disease on repeat CT scans, as well as endoscopy, was found. The patient underwent craniotomy and complete excision. Pathologic analysis confirmed a poorly differentiated adenocarcinoma with marked necrosis consistent with a metastasis from the primary esophageal neoplasm. He was discharged on the third postoperative day without residual hemiparesis and received adjuvant whole-brain radiation therapy beginning 3 weeks postoperatively (3375 cGy in 15 fractions). He has been carefully followed with CT imaging and endoscopy on a routine basis. The patient remains alive and well 6 years after esophagogastrectomy and 5.5 years after excision of the brain metastasis.

\section{Discussion}

Several treatment options exist for symptomatic brain metastases, including whole-brain radiation, stereotactic radiation, and surgical intervention, which can be used alone or in combination. Because prospective clinical trials are lacking, treatment selection has been individualized on the basis of a number of variables, including tumor location, tumor size, number of CNS metastases, functional status, presence or absence of extracranial disease, and institutional biases. Two recent retrospective studies of patients with esophageal cancer with CNS metastases and mainly adenocarcinoma histology have been published. Weinberg and colleagues ${ }^{1}$ reviewed 27 patients from M.D. Anderson Hospital. The median survival after the diagnosis of a CNS metastasis was 3.8 months. The longest survival was 9.6 months, which occurred in one of the 4 patients who were treated with surgical intervention and whole-brain radiation. Khuntia and associoates ${ }^{2}$ reported the Cleveland Clinic experience with 27 patients, of whom 7 were selected to undergo surgical intervention. In 5 of these patients, surgical intervention was used in combination with whole-brain radiation. The median survival was 3.6 months; however, 1 patient survived for 48 months. Survival in both series appeared to be improved with aggressive therapy, which included surgical intervention, offered only to a select subset of patients.

Mao and coworkers ${ }^{3}$ reported a patient with a clinical stage II adenocarcinoma of the esophagus treated with neoadjuvant chemoradiation therapy followed by esophagogastrectomy. An isolated CNS metastasis was treated with surgical intervention and whole-brain radiation 18 months later. The patient had survived more than 7 years at the time of the report. In a recent study from our institution, ${ }^{4}$ a cohort of 176 patients surgically treated for locally advanced adenocarcinoma was analyzed. Further analysis of these data identified 4 (2.3\%) patients who were subsequently found to have CNS metastases. Three of these patients had re- 

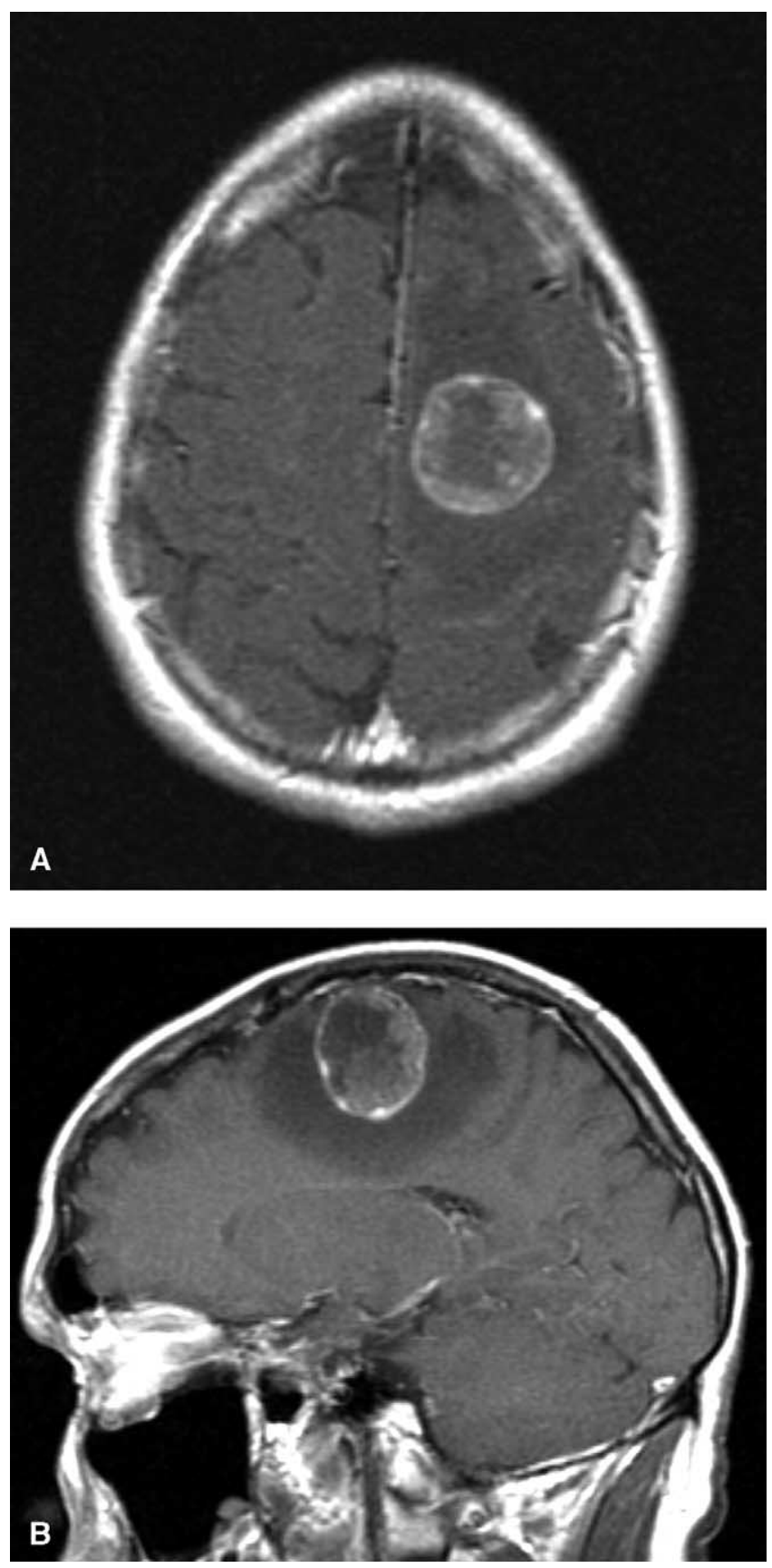

Figure 1. A, Magnetic resonance imaging: axial T1-weighted postcontrast image. B, Sagittal T1-weighted postcontrast image. ceived chemoradiation therapy before esophagogastrectomy, and the other underwent surgical intervention without chemoradiation therapy. Two patients, including the patient in this report, demonstrated an isolated CNS metastasis without evidence of other disease. The other 3 patients were treated with radiation and died after an average of 6 months. We believe our patient and the patient previously reported demonstrated several variables that might be predictive of prolonged survival, including a good performance status, complete or near-complete pathologic response to neoadjuvant chemoradiation, and isolated lesions amenable to complete surgical excision.

\section{Conclusion}

Reported outcomes of patients with esophageal cancer with CNS metastases have been dismal. Previous retrospective series, however, have been small and included a relatively heterogeneous group of patients with respect to several prognostic variables, and most patients have received nonsurgical treatment. CNS metastases after treatment of esophageal cancer will likely become more frequent because the incidence of adenocarcinoma is increasing and systemic (versus local) recurrence is becoming more common with the escalating use of neoadjuvant chemoradiation therapy. We believe that carefully selected patients have the potential to experience long-term survival and therefore should be treated aggressively.

\section{References}

1. Weinberg JS, Suki D, Hanbali F, et al. Metastasis of esophageal carcinoma to the brain. Cancer. 2002;94:759-64.

2. Khuntia D, Sajja R, Chidel MA, et al. Factors associated with improved survival in patients with brain metastases from esophageal cancer: a retrospective review. Technol Cancer Res Treat. 2003;2:267-72.

3. Mao YS, Suntharalingam M, Krasna MJ. Management of late distant metastases after trimodality therapy for esophageal cancer. Ann Thorac Surg. 2003;76:1742-3.

4. Kesler KA, Helft PR, Werner EA. A retrospective analysis of locally advanced esophageal cancer patients treated with neoadjuvant chemoradiation therapy followed by surgery or surgery alone. Ann Thorac Surg. 2005;79:1116-21. 